Relations industrielles

Industrial Relations

\title{
Groarke, Leo (sous la direction de), The Ethics of the New
}

Economy

\section{Fernand Morin}

Volume 54, numéro 1, 1999

Relations industrielles et nouveaux systèmes productifs

Industrial Relations in the New Workplace

URI : https://id.erudit.org/iderudit/051226ar

DOI : https://doi.org/10.7202/051226ar

Aller au sommaire du numéro

Éditeur(s)

Département des relations industrielles de l'Université Laval

\section{ISSN}

0034-379X (imprimé)

1703-8138 (numérique)

Découvrir la revue

Citer ce compte rendu

Morin, F. (1999). Compte rendu de [Groarke, Leo (sous la direction de), The Ethics of the New Economy]. Relations industrielles / Industrial Relations, 54(1), 197-201. https://doi.org/10.7202/051226ar

Tous droits réservés (C) Département des relations industrielles de l'Université Laval, 1999
Ce document est protégé par la loi sur le droit d'auteur. L'utilisation des services d'Érudit (y compris la reproduction) est assujettie à sa politique d'utilisation que vous pouvez consulter en ligne.

https://apropos.erudit.org/fr/usagers/politique-dutilisation/ 


\section{Recensions}

\section{Book Reviews}

\section{The Ethics of the New Economy}

collectif sous la direction de Leo GROARKE, Waterloo, Ont. : Wilfrid Laurier University Press, 1998, 332 p., ISBN 0-88920-311-3.

En raison de la diversité des sousthèmes étudiés, le lecteur voudra savoir ce que l'on entend à la fois par ces deux mots clés du titre rassembleur: éthique et économie nouvelle. D'entrée de jeu, le professeur Leo Groarke souligne à grands traits les caractéristiques de cette nouvelle économie à l'aide de ses principales manifestations : fermeture d'établissement, fusion, réorganisation du travail, licenciement collectif, robotisation, sous-traitance, etc. Les conséquences de semblables soubresauts peuvent être parfois bénéfiques à certains, mais elles sont trop souvent pénibles à tant d'autres. L'ouvrage propose de multiples analyses sur ce thème, notamment par la discussion du choix des moyens retenus. Ces contributions permettent au lecteur d'acquérir une compréhension plus approfondie de la problématique éthique que soulève cette réforme de l'économie. À ces fins, on y retrouve des éléments de réponse à ces questions : À quelles conditions une réorganisation radicale de l'entreprise seraitelle nécessaire? Quelles sont alors les obligations des gestionnaires envers les salariés? Quel enseignement pouvonsnous tirer des réorganisations déjà réalisées? Quels sont les principaux aspects éthiques considérés? Est-ce que ces opérations à hauts risques peuvent servir au développement d'un comportement éthique de la part des gestionnaires? etc.

Selon le directeur Groarke, cet ouvrage portant sur l'éthique pratique traite des valeurs susceptibles d'être retenues par les intéressés, tout en considérant l'ensemble des données factuelles constituant chaque situation et, notamment, celles d'ordre social, historique, politique, technique, etc. À ces fins, l'éthique pratique exige une analyse certaine et vaste de l'ensemble des composantes d'une question et une telle démarche permettrait à une personne responsable et respectueuse des autres de prendre la meilleure décision. On le constate déjà, un tel entendement de l'éthique signifie que la question de la réorganisation d'une entreprise doit se faire en considérant ses implications pour tous les partenaires réels de l'entreprise : les actionnaires, les salariés, les fournisseurs, les clients ou usagers et la communauté. Cette approche pratique de l'éthique suppose une étude interdisciplinaire et tel est d'ailleurs le trait caractéristique de cet ouvrage collectif dont le contenu est ainsi résumé : «By including articles that promote responsible restructuring along with others that more universally reject restructuring, the book tries to present an informed account of different sides of the restructuring debate » (p. 7). Bien évidemment, chaque auteur ne pouvait offrir une analyse de toutes les facettes de la question retenue. L'ensemble des exposés permet au lecteur de mieux saisir la complexité de la question principale et d'entrevoir les meilleures voies à suivre pour effectuer une réorganisation d'une entreprise, si toutefois elle s'impose, toutes choses bien considérées.

Ce collectif comprend neuf chapitres regroupant 24 articles rédigés par 31 
auteurs. Le plan de l'ouvrage est établi de manière à y traiter successivement de l'entreprise privée et publique, des avantages et inconvénients de la réorganisation, de la responsabilité des acteurs, des méthodes de réalisation et de leurs effets sur l'emploi, auprès des concurrents et du milieu social. Finalement, on procède à l'étude de dossiers particuliers ou à plus forte teneur politique: l'économie du Nouveau-Brunswick, l'éducation en Alberta, la réorganisation de la compagnie Bell et du secteur bancaire. L'ouvrage offre une intéressante bibliographie (p. 309 à 327 ) dont près de $30 \%$ est de source américaine. L'obstacle de la langue, sans doute, n'aurait pas permis aux 31 auteurs de référer à des sources québécoises telles la revue Ethica, les Cahiers de recherches éthiques, etc.

Ne pouvant commenter distinctement ces 24 articles, nous ne soulignons que les textes qui nous semblent les plus intéressants sachant qu'il n'y a pas de méthodes plus subjectives que celle que nous empruntons. Quiconque pourrait allègrement contester ce premier choix en tenant compte des circonstances de temps et de lieu différentes des nôtres. Une "question préalable " pouvait être soulevée en raison des qualités professionnelles des auteurs et de l'éditeur, soit celle de la pertinence que des universitaires traitent pareils sujets pratiques. Le doyen Scott Carson (p. 9 à 25) répond avec brio à cette question, à savoir qu'il s'agit de l'organisation de la vie économique, sociale et politique du pays et que l'université a le devoir de participer à ce débat et d'offrir des perspectives à long terme qui seraient socialement justes. En somme, la définition mythique et irréaliste de ce que devrait être l'université et ses professeurs ne serait qu'un leurre et nullement un obstacle véritable à la nécessaire contribution des universitaires à ces difficiles et cruciales questions qui demandent une analyse interdisciplinaire réalisée par des personnes libres. Le doyen Carson reconnait cependant que le professeur qui œeuvre trop près des gestionnaires à titre de conseil, ou autrement, pourrait ne pas disposer de la liberté nécessaire à de telles analyses impartiales et générales.

Le professeur Barry Hoffmaster (p. 25 à 40) offre une belle analyse critique de cette vague de fond qui pousse tant de gestionnaires à imposer des réorganisations profondes des processus de production de biens et de services. L'auteur soumet que l'approche éthique de la réorganisation des entreprises ne peut être contenue à la simple notion de la conscience individuelle ou à celle du respect des lois face aux contraintes inexorables de la mondialisation. Les implications sociales, économiques et politiques de ces changements imposeraient de retenir une approche plus globale et plus interdisciplinaire. Pour mieux illustrer ce point de vue, Hoffmaster souligne l'interrelation des intérêts de nombreuses entreprises à ceux des administrateurs de fonds de retraite qui sont la propriété de milliers de salariés ou d'ex-salariés. Au bénéfice de ces derniers, on ne saurait faire pression auprès des gestionnaires à l'aide du bilan trimestriel et, en même temps, vouloir que ces mêmes gestionnaires sachent retenir une approche à long terme à l'égard des salariés actuels. À cette fin, l'auteur rappelle le principe de l'altérité, "them is us " ou notre inéluctable interdépendance. Ce principe impose une approche responsable au sens profond du terme et nécessairement fort plus large que son entendement juridique. Pour mieux illustrer son propos, le professeur Hoffmaster analyse le processus retenu en Ontario devant permettre une restructuration des services de santé. Malgré le double objectif déclaré, soit la réduction du déficit et l'amélioration des services, l'auteur explique que le premier fut atteint au détriment du deuxième. Ce dernier n'aurait servi que d'enrobage oratoire. Ce dossier relatif aux services de santé sert aussi à expliquer que l'on ne peut retenir le programme T.Q.M. (Total Quality Management) en imposant des réformes d'autorité ou par 
voie descendante et mu par le seul souci de l'efficacité comptable: "The central moral issue here is an old one about trade-offs between efficiency and effectiveness, quantity and quality " (p. 34). Lorsque le bénéficiaire des services de santé est dénommé client et non plus patient, on ne peut que constater que le langage véhicule bien l'esprit mercantile sous-jacent à cette réforme (p. 38). Quant à l'usage de techniques de gestion telle que la qualité totale pour les fins de l'administration des services de santé, d'éducation et autres, il existerait un danger de glisser rapidement dans une approche matérialiste où l'homme a peu à dire et surtout, à recevoir. Ainsi, la question éthique doit être soulevée et traitée pour éviter la conversion de ces services en de simples biens de consommation.

À l'aide de quatre exemples, le professeur Cascio indique comment on peut effectuer ces opérations de grande envergure d'une façon à la fois efficace et respectueuse des salariés et du milieu social. L'auteur souligne qu'un changement aussi radical doit être fait par " beau temps " et non en période de crise et qu'il devrait être réalisé avec les salariés, d'où l'importance de la mise en place d'un réseau de communication qui serait multi-directionnel. Soulignant les expériences de Ford, Chevron, 3M et Avon, l'auteur montre bien qu'un bon programme de formation professionnelle peut permettre notamment d'être "clean, lean without being mean ». Finalement, le professeur Cascio formule 12 recommandations susceptibles de permettre une restructuration qui serait à la fois valable pour tous: them is us (p. 72).

L'article intitulé "Corporate Judo" (p. 75 à 92) souligne les risques qu'encourt toute entreprise qui annonce prématurément un licenciement massif, une fusion ou une restructuration. Elle peut dès lors devenir la proie des concurrents et il serait même possible que bien des actionnaires, des employés et des clients la délaissent aussitôt. De tels départs ne pourraient qu'accentuer la fragilité de l'aventure. Dès lors, les auteurs formulent une théorie qui suppose de la part des instigateurs d'un tel renouveau de savoir tirer avantage de la situation en prévenant les réactions prédatrices des tiers : vigilance, prudence et sagesse. Le septième article traite d'un épiphénomène résultant de la situation financière critique que connaissent maintes institutions publiques et qui sont à la recherche de nouvelles sources de financement par voie d'une commandite commerciale ("CauseRelated Marketing ", par P. Cunningham et P.J. Cushing, p. 95 à 109). La problématique éthique est intéressante puisque ces deux partenaires d'occasion répondent de finalités fort différentes, voire opposées (la santé ou l'éducation pour les uns, la vente et autres aspects commerciaux pour les autres). On saisit bien que la relation philanthropique du commanditaire est souvent inexistante ou elle n'est qu'apparente. Une telle union de raison exige de la part des instigateurs la clairvoyance des embûches éventuelles, le respect des sphères de compétences respectives et une franchise indéfectible. Tel serait, rappelle-t-on, le défi constant (avant, pendant et après l'union) que doivent savoir relever les deux équipes de gestionnaires d'un tel partenariat.

Le professeur Vince Di Norcia ("Downsizing, Change and Ownership ", p. 143 à 154) illustre de multiples façons qu'une réduction drastique du personnel doit s'effectuer avec les salariés visés et d'une façon franche. On ne saurait, d'une part, prétendre qu'il s'agit de la "force vive de l'entreprise " et, d'autre part, traiter ces salariés comme de simples biens de consommation. Si, dans l'intérêt des actionnaires, des gestionnaires et des salariés, il peut être parfois nécessaire de procéder à un élagage ou à un redéploiement des ressources, des programmes de formation professionnelle permettant une modernisation ou un ajout de créneaux sont préférables et plus utiles que d'entreprendre un simple abattage. En considérant comment des gestionnaires de grandes entreprises réalisèrent aussi 
ouvertement et rapidement des licenciements collectifs pour s'assurer une bonne fin de trimcstre, Di Norcia souligne que le pouvoir de direction de l'entreprise repose encore sur un système juridique et politique anachronique où toute l'autorité résulte des titres de propriété (p. 150). Compte tenu des intérêts interdépendants en présence, ce rapport pouvoir/propriété devrait être modifié, soutient-il, de manière à ce que le personnel d'une entreprise puisse participer à ces décisions comme c'est déjà le cas notamment en Allemagne. À ce sujet, l'auteur rappelle l'histoire ontarienne de l'entreprise Algoma Steel où la participation des réels partenaires de l'entreprise lui fut salutaire (p. 153). Il pourrait être intéressant de savoir lesquels des financiers ou des gestionnaires seraient les plus opposés à une semblable réforme de l'entreprise?

Dans un autre article tout aussi vivifiant, David Drinkwalter ("Discharging Employer Responsibilities to Employees during Major Organizational Change", p. 167 à 177) insiste sur la formation professionnelle comme voie de solution qui serait à la fois positive, dynamique et mutuellement avantageuse. En d'autres termes, cette approche s'autoriserait d'une décision éthique puisqu'elle considère à la fois les interrêts de tous et de chacun. Pour éviter le syndrome du survivant, il importerait, rappelle-t-il, que les salariés qui conservent leur emploi puissent croire et avoir raison de le faire et que ceux qui durent quitter furent honnêtement et équitablement traités.

La dernière partie de l'ouvrage présente cinq articles où on y traite autant de situations distinctes, mais néanmoins réunies par un même intérêt partagé, celui de l'étude des implications sociales et éthiques de ces restructurations. Cette diversité des problématiques enrichit valablement nos connaissances et, notamment, en nous faisant mieux saisir la nécessité de rechercher, en chaque cas, les voies de solution les plus appropriées. Une démarche valable en milieu scolaire ou de la santé peut être une erreur ailleurs.
Le dernier article de l'ouvrage fut confié au professeur Wesley Cragg et constitue une note terminale à la question relative au possible apport de l'éthique à cette problématique de la réorganisation des entreprises. L'auteur indique, en premier lieu, les entendements divers ou opposés que l'on peut entretenir à l'égard de l'éthique. Il note que certains croient encore que la préoccupation éthique devrait relever plus de la famille et de l'école que de la gestion des affaires. Avec raison, croyons-nous, le professeur Cragg souligne que l'éthique ne produit pas d'effets magiques ni nécessairement bénéfiques. Par ailleurs, l'éthique pousse le décideur à ouvrir plus grandes ses considérations à la fois par une analyse des effets de ses décisions possibles à l'égard des autres (them is us) et à long terme. Le professeur Cragg souligne qu'au lieu de traiter de la question éthique par voie de jugement critique sur les négligences graves de certaines entreprises, il serait plus valable d'analyser comment certains gestionnaires ont pu valablement élaborer puis réaliser un processus de restructuration qui respecte les valeurs éthiques prévalantes. En somme, on ne saurait aborder l'éthique pour semoncer ni se limiter à un discours moralisateur alors que l'éthique serait, comme le juste, un fait et c'est à ce niveau finalement que l'on doit s'attarder pour saisir et apprécier les qualités des décisions prises.

Ces vingt-quatre articles permettent de mieux saisir la diversité des processus retenus pour négocier ces adaptations radicales des entreprises à la "nouvelle économie *. Entre l'ablation, c'est-à-dire les licenciements collectifs brusques et tapageurs et le renouveau d'une entreprise réalisé avec les salariés, l'écart peut être énorme et pour les personnes en présence et pour l'économie du pays. Lorsque la destruction, le démantèlement d'une organisation devient une fin et qu'elle ne donne pas prise à un nouveau départ, le bilan ne peut être que négatif sauf pour quelques "héritiers " parmi les actionnaires et les gestionnaires. Plusieurs 
auteurs ont éloquemment illustré l'importance de la communication entre toutes les parties nécessairement tributaires de l'opération. L'information donnée d'une façon claire, honnête et continuelle permet d'éviter des réactions vives de certains, d'éliminer les rumeurs dévastatrices et, surtout, elle manifeste un réel respect des autres. Ces mêmes auteurs signalent également comment de véritables programmes de formation professionnelle peuvent être salutaires et pour les salariés et pour les entreprises. En somme, ce temps de formation à une période de transition peut faciliter l'attente du nouveau départ et permettre à l'entreprise de disposer d'une maind'œuvre qualifiée, fidèle et dévouée. Outre le respect de tous les véritables partenaires de l'entreprise et de l'importance de la prise d'une décision d'une manière responsable, les auteurs n'ont guère traité directement de l'éthique. Ce volet est abordé davantage par voie d'illustrations de ce qu'est et de ce que ne pourrait être une décision d'un gestionnaire respectueuse des intérêts de tous (actionnaires, gestionnaires, salariés, clients, fournisseurs et société), toutes choses bien considérées. En d'autres termes, on a préféré l'évidence tirée de l'expérience à la remontrance moralisatrice. Il nous serait difficile de contester ce choix.

FERNAND MORIN

Université Laval

\section{Researching the World of Work - Strategies and Methods in Studying Industrial Relations}

publié sous la direction de Keith WHITFIELD et George STRAUSS, New York: Cornell University Press, 1998, 330 p., ISBN 0-8014-8549-5.

Cet ouvrage vise à exposer et à questionner les stratégies de recherche et les méthodes utilisées en relations industrielles. Le public cible s'avère les chercheurs, les praticiens et les étudiants qui souhaitent mieux comprendre l'évolution de la recherche et des études en relations industrielles et les choix méthodologiques qui s'offrent à eux pour en approfondir la compréhension. Il s'agit d'un collectif où 22 auteurs, mandatés par le Research Methods Study Group of the International Industrial Relations Association, fournissent un tour d'horizon plutôt exhaustif et dynamique de ce qui s'est fait et de ce qui peut être fait dans l'étude empirique des relations industrielles. Au début du livre, les directeurs expliquent que la nécessité de procéder à la publication de cet ouvrage provient du constat suivant : jusqu'à récemment la plupart des recherches en relations industrielles privilégiaient, en grande partie, l'étude des relations patronales-syndicales mais, depuis quelques années, le spectre s'élargit autour des enjeux de performance du monde du travail laquelle inclut la santé et la sécurité du travail, les pratiques managériales, la discrimination, la satisfaction des employés, la sécurité d'emploi et l'analyse comparative des systèmes de relations industrielles. Ces thèmes ne sont pas nouveaux certes, mais leur étude a généré encore plus d'interactions avec des chercheurs d'autres disciplines. De plus, les auteurs constatent une plus grande variété de stratégies et de méthodes utilisées pour répondre aux questions de recherche du monde contemporain des relations industrielles.

Bien que ce livre puisse se voir attribuer l'étiquette de théorique, il ne discute pas de forces ou de faiblesses de modèles théoriques utilisés en relations industrielles, il ne tient pas non plus à faire de débat sur les relations industrielles en tant que champ ou discipline. Cependant, un puissant fil conducteur tisse des liens solides entre les chapitres: la recherche empirique, soit celle qui repose sur l'étude de faits et ce, qu'il s'agisse du champ d'étude ou d'une discipline particulière au sein des relations industrielles. 